\title{
Personality from a controlled processing perspective: An fMRI study of neuroticism, extraversion, and self-consciousness
}

\author{
NAOMI I. EISENBERGER, MATTHEW D. LIEBERMAN, and AJAY B. SATPUTE \\ University of California, Los Angeles, California
}

\begin{abstract}
Although neuroticism has been central to most theories of personality, self-reported neuroticism has had limited success in predicting expected behavioral outcomes. The reason for this may be due, in part, to the imprecision of self-reports. The purpose of this study was to examine the relationship between neural correlates of control systems and neuroticism, extraversion, and self-consciousness. In response to an oddball task, neuroticism was associated with increased dorsal anterior cingulate cortex (dACC) reactivity, typically associated with discrepancy detection, whereas extraversion and selfconsciousness were associated with lateral and medial frontoparietal networks, respectively, typically associated with task-focused (lateral) or self-focused (medial) controlled processes. We also examined whether the neural measure of neuroticism would predict a relevant behavioral outcome better than self-reports would. Interoceptive accuracy, an outcome associated with neuroticism, was better accounted for by dACC reactivity $\left(r^{2}=.74\right)$ than by self-reported neuroticism $\left(r^{2}=.16\right)$, suggesting that neural reactivities may provide a more direct measure of personality than self-reports do.
\end{abstract}

Neuroticism, a trait characterized by the tendency to experience negative affect (Costa \& McCrae, 1980; H. J. Eysenck, 1967), appears in nearly all taxonomies of personality. Despite neuroticism's centrality to personality theories, self-report measures of neuroticism have had limited success in predicting expected behavioral outcomes in the areas of mood, conditioning, attention, and vigilance (Matthews \& Gilliland, 1999). Why should such a well-recognized psychological dimension, which has become a common descriptor of personality by researchers and laypersons alike, have such limited predictive ability when the outcomes it is intended to predict are examined? We believe that multiple issues related to self-report techniques are partly responsible and that such tools as functional magnetic resonance imaging (fMRI) can help to improve the study of neuroticism, as well as other personality variables.

Although self-report measures have been the primary means of assessing personality constructs and have

This research was graciously funded by Grant R21MH66709-01 from the National Institute of Mental Health to M.D.L. For their generous support, the authors also thank the Brain Mapping Medical Research Organization, the Brain Mapping Support Foundation, the PiersonLovelace Foundation, the Ahmanson Foundation, the Tamkin Foundation, the Jennifer Jones-Simon Foundation, the Capital Group Companies Charitable Foundation, the Robson Family, the William M. and Linda R. Dietel Philanthropic Fund at the Northern Piedmont Community Foundation, the Northstar Fund, and the National Center for Research Resources (Grants RR12169, RR13642, and RR08655). Correspondence concerning this article should be addressed to N. I. Eisen- berger or M. D. Lieberman, Department of Psychology, University of California, Franz Hall, Los Angeles, CA 90095-1563 (e-mail: neisenbe@ ucla.edu or lieber@ucla.edu). served personality researchers well, self-report measures have also been criticized for being flawed and inaccurate (Nisbett \& Wilson, 1977). Given that two of the most prominent personality theories (H. J. Eysenck, 1967; J. A. Gray, 1981, 1982) have posited that neuroticism, or the related personality construct of anxiety, relates to individual differences in the reactivity of underlying neural structures that process threatening information, it makes sense that self-report measures have been insufficient, for several reasons. First, self-report measures of neuroticism assess the affective consequences of threat detection (e.g., "Are your feelings easily hurt?" "Does your mood often go up and down?"), rather than the threat detection mechanisms themselves. Second, even if self-report measures did assess threat detection mechanisms, individuals may lack introspective access to this type of information. Thus, although individuals may have introspective access to their final affective state, they may not be aware of the processes that led to that state (Nisbett \& Wilson, 1977; E. R. Smith \& Miller, 1978). Consequently, self-report measures may model a phenomenological endpoint that is related to, but not the same as, the neurocognitive mechanisms that drive behavior, affect, and cognition. In other words, affective phenomenology may be an index of how a neuroticism-related mechanism is functioning; the affect may be a consequence, rather than a core component, of neuroticism and, thus, may be a noisy output signal rather than a true reflection of neuroticism.

If these secondary affective consequences are not the core components of neuroticism, what are? According to one prominent theory of anxiety ${ }^{1}$ (J. A. Gray, 1981, 1982), high levels of anxiety are due to a more sensitive neural comparator, a putative mechanism that detects mis- 
matches between the actual and the expected states of the world, allowing an organism to modulate its behavior to attain or avoid certain stimuli. In line with appraisal models of emotion, this detection of discrepancy between the actual and expected states of the world is what triggers the negative affect (Carver \& Scheier, 1990; Lazarus, 1991; Mandler, 1975) that is so often associated with neuroticism. In light of these models, neuroticism might be defined, at least in part, as a hypersensitivity to discrepancy, which can then lead to negative affect as one of the manifesting consequences.

Controlled processing mechanisms, which typically include a discrepancy detection component, have been influential in the study of emotion, cognition, and social psychology. In the next section, we will review the core features of these mechanisms, because they provide a framework for investigating neuroticism, as well as other personality dimensions. In addition, some of the neural correlates of controlled processing mechanisms have been well mapped in the past decade and thus provide a starting point for linking personality to neurocognitive differences. We suggest that the reactivity of the dorsal anterior cingulate cortex (dACC) is directly related to neuroticism, due to its role in discrepancy detection, whereas the reactivity of neural regions involved in other aspects of controlled processing are more closely related to extraversion and self-consciousness. Specifically, we suggest that extraversion is related to increased activity in the lateral frontoparietal network, typically associated with task-focused controlled processing, whereas selfconsciousness is related to increased activity in the medial frontoparietal network, typically associated with self-focused controlled processing.

\section{Control Systems and Their Neural Correlates}

Both cognitive and social psychologists have borrowed from machine-based models of self-regulating systems to understand how individuals regulate behavior and reach important goals. At the core of these self-regulating systems is the TOTE unit, an acronym referring to the process of testing for discrepancies from a desired set point or goal, operating on the environment in order to reduce these discrepancies, testing for discrepancies once again, and exiting if none remain (Miller, Galanter, \& Pribram, 1960; Weiner, 1948). Psychological TOTE units are thought to function in much the same way that a thermostat does. Just as a thermostat "self-regulates" by detecting discrepancies from a preset temperature and turning on warm or cool air to change the temperature of a room, so too can individuals self-regulate by detecting discrepancies from relevant goals and enacting cognitive strategies or behaviors to reduce these discrepancies.

Cognitive psychologists have used TOTE units to understand how individuals complete novel or difficult tasks (Shallice, 1988). These models highlight the importance of discrepancy detection mechanisms for identifying incorrect responses and of working memory sub- strates for external or task-focused processing aimed at overriding incorrect responses. Social psychologists, on the other hand, have used TOTE units to understand how individuals self-regulate (Carver \& Scheier, 1981; Duval \& Wicklund, 1972; Higgins, 1987; Vallacher \& Wegner, 1987). These models emphasize the use of discrepancy detection mechanisms for noting when one is not attaining important goals and of internal or self-focused processing for understanding why such a discrepancy has occurred ("Why am I not succeeding at this?") and for evaluating one's general capacities for the task at hand ("I am no good at this sort of thing"). These models suggest that self-focused attention can have a variety of effects on cognition and behavior, including generating alternative behavioral responses, developing more global plans that will affect future behaviors, or ruminating about why one is not attaining one's goal.

In the past decade, models of cognitive control have been grounded within a cognitive neuroscience framework. Cohen and colleagues have posited that the dACC plays a role in discrepancy detection (Botvinick, Braver, Barch, Carter, \& Cohen, 2001; Carter et al., 1998; Carter et al., 2000), acting as the test unit or trigger for cognitive control. Across several neuroimaging and computational modeling studies, it has been shown that the dACC is activated by certain types of discrepancies-for example, behavioral response conflicts such as those produced in the Stroop task (Botvinick et al., 2001). In addition, the dACC may be sensitive to goal conflicts, to expectation violations, and to errors more generally (Braver, Barch, Gray, Molfese, \& Snyder, 2001; Weissman, Giesbrecht, Song, Mangun, \& Woldorff, 2003). Indeed, it has been suggested that the dACC may respond to discrepancies at various levels of information processing, including the level of stimulus evaluation and task representation (Botvinick, Cohen, \& Carter, 2004) and, possibly, the level of goal pursuit.

Following the detection of discrepancy, the lateral prefrontal cortex (LPFC), which plays a role in working memory (E. E. Smith \& Jonides, 1999) and decision-making processes (Bunge, Hazeltine, Scanlon, Rosen, \& Gabrieli, 2002), acts as the operate unit, instantiating cognitive control by biasing responses in a goal-congruent manner (Botvinick et al., 2001). In addition, the lateral posterior parietal cortex (LPPC) is another region that appears to be involved in controlled processing, as evidenced by its activation during controlled processing tasks and its role in working memory processes that are necessary for the instantiation of cognitive control (Ardekani et al., 2002; Braver et al., 2001; Bunge et al., 2002; Weissman et al., 2003).

The social psychological models linking TOTE units with self-regulation and self-focused attention have not yet been examined from a cognitive neuroscience perspective. However, studies of self-focused attention have observed activity in the $\mathrm{dACC}$, as well as in the medial prefrontal cortex (MPFC) and the precuneus within the 
medial posterior parietal cortex (MPPC; Gusnard, Akbudak, Shulman, \& Raichle, 2001; Johnson et al., 2002; Kampe, Frith, \& Frith, 2003; Kircher et al., 2001; Kjaer, Nowak, \& Lou, 2002). Thus, it is possible that cognitive control and self-control share a common test process, subserved in part by the dACC, but that this trigger can lead to external or task-focused controlled processes in lateral cortical regions, whereas this same trigger can lead to internal or self-focused controlled processes in medial cortical regions (Ochsner et al., 2004).

\section{Control Mechanisms and Personality}

Given the above neural characterization of discrepancy detection mechanisms and both externally and internally focused control processes, it is possible to hypothesize how individual differences in the functioning of these neural mechanisms might relate to or be a core component of a number of personality dimensions. First and foremost, we hypothesized that neuroticism would correspond to dACC reactivity to the presence of a discrepancy, so that greater reactivity in the dACC to an oddball task involving discrepancy detection would be associated with greater levels of neuroticism. In line with this, individuals with obsessive-compulsive disorder, one type of anxiety disorder, have shown increased dACC activity to a task involving response conflict (Ursu, Stenger, Shear, Jones, \& Carter, 2003). In addition, heightened dACC activity has been found in anxiety-disordered patients (Breiter et al., 1996; Bystritsky et al., 2001) and has been found to be associated with symptoms of anxiety in both patients (Osuch et al., 2000) and healthy adults more generally (Kimbrell et al., 1999). We also investigated whether neuroticism would be negatively correlated with rostral ACC (rACC) activity, because two studies have shown that neuroticism-related measures correlated negatively with rACC activity (J. R. Gray \& Braver, 2002; Bishop, Duncan, Brett, \& Lawrence, 2004). We did not have strong predictions about the relation between neuroticism and either of the frontoparietal networks; however, given previous studies showing positive correlations between neuroticism and self-consciousness (e.g., Trapnell \& Campbell, 1999), a positive correlation between neuroticism and the medial frontoparietal network seemed plausible.

Second, we hypothesized that extraversion, a personality dimension associated with externally focused attention, enhanced working memory capacity, and greater LPFC activity to a working memory task (Kumari, ffytche, Williams, \& Gray, 2004; Lieberman, 2000; Lieberman \& Rosenthal, 2001), would be associated with greater reactivity in the LPFC and LPPC in response to discrepancy. Thus, we predicted that extraverts would respond to discrepancy by exerting control over the external task environment, which recruits the lateral frontoparietal network, rather than by focusing on themselves, which recruits the medial frontoparietal network. Finally, we hypothesized that self-consciousness, a personality dimension characterized by a tendency to engage in self- focused attention, would be associated with greater reactivity in the MPFC and MPPC in response to discrepancy. This prediction was based on the assumption that self-conscious individuals respond to discrepancy detection by focusing on themselves, rather than on the external task environment, a process that recruits the medial frontoparietal network, rather than the lateral frontoparietal network.

\section{Assessing Neural Reactivities}

To assess these neural reactivities, participants performed an oddball task while undergoing fMRI scans. The oddball task is a discrepancy detection task in which the participant is told that most of the time he or she will see the letter $X$ appear on a computer screen but that every so often he or she will see a different letter (oddball) and that he or she should respond to these infrequent presentations. The relevant discrepancy in the oddball task is between the participant's baseline expectation, which is to see mostly Xs, and the presentation of a non-X letter (the oddball trial). Previous studies have shown that the oddball trials, as compared with the nonoddball trials, reliably produce dACC activity (Ardekani et al., 2002; Braver et al., 2001; Weissman et al., 2003). Although the oddball task does not seem to require a great deal of top-down control, LPFC and LPPC activations have also been observed in these studies (Ardekani et al., 2002; Braver et al., 2001; Weissman et al., 2003).

Using this task has significant advantages for the assessment of the neural correlates of personality, particularly for neuroticism and self-consciousness. First, we have hypothesized that neuroticism is related to discrepancy detection, a mechanistic precursor of negative affect, rather than to the experience of negative affect per se. In daily life, discrepancy detection and negative affect are probably confounded during most episodes. However, the oddball task is so minimal in its demands and its implications for self-evaluation that it is not particularly distressing and, thus, allows discrepancy detection to be assessed without the affective consequences seen with more meaningful types of discrepancies. Second, we have hypothesized that the MPFC and MPPC will be activated during the oddball task to the extent that individuals are high in self-consciousness. These are not brain regions typically observed in the oddball task, but if they are present, this may represent a personality-specific consequence of dACC activation that falls in line with social psychological theories of self-focused attention.

\section{Assessing a Behavioral Correlate of Neuroticism}

It would make little sense to build personality models from scratch, using neurocognitive measures without reference to the self-report measures that have been in use for decades. Thus, in most of the analyses in the present work, relationships between task-specific neural reactivities and scores on self-report measures will be examined. Still, because self-report measures have certain weaknesses, it is important to do more than examine 
correlations between neural activity and self-report measures. One way to proceed is to examine the extent to which personality-related neural activity predicts behavioral outcomes that the personality dimension should predict. If the personality-related neural activity predicts the behavior better than the self-report measures do, this suggests that the personality-related neural activity may be a more direct measure of the personality dimension than the self-report measures are.

For the present study, we chose to examine interoceptive accuracy, the awareness of changes in physiological arousal levels, as a behavioral outcome measure that is relevant to neuroticism. There were several reasons for this choice. First, and consistent with lay conceptions of neuroticism, multiple studies have shown that neurotics and those who score high on the related construct of trait anxiety or trait negative affectivity tend to report more somatic symptoms and to show an increased attention to physiological changes (Watson \& Pennebaker, 1989). Neurotics' enhanced reporting of physiological sensations seems to reflect a greater sensitivity to internal changes, rather than a greater physiological reactivity (Asmundson, Norton, Wilson, \& Sandler, 1994; Schandry, 1981; Shostak \& Peterson, 1990; Stewart, Buffett-Jerrott, \& Kokaram, 2001). Several studies have shown that individuals who score higher on neuroticism-related personality measures show greater interoceptive awareness of physiological changes than those who score lower on these measures do (Critchley, Wiens, Rothstein, Ohman, \& Dolan, 2004; Ehlers \& Breuer, 1992; Ludwick-Rosenthal \& Neufeld, 1985; Schandry, 1981; Stegen, Van Diest, Van de Woestijne, \& Van den Bergh, 2001; Stewart et al., 2001; Sturges \& Goetsch, 1996). Second, changes in physiological arousal levels represent a discrepancy between the normal state of the body and a more aroused state. Thus, individuals with more sensitive discrepancy detection mechanisms should be more cognizant of such changes. Lastly, detection of changes in arousal may serve as an important cue to a threat that ought to be detected by the neural circuitry under investigation. Thus, we hypothesized that self-reported neuroticism would be associated with dACC activity but that dACC activity would be a better predictor of interoceptive accuracy than self-reported neuroticism would, because dACC reactivity is a more direct assessment of the neurocognitive mechanisms underlying neuroticism.

\section{METHOD}

\section{Participants}

The participants $(n=17)$ were healthy, right-handed individuals recruited from the University of California, Los Angeles (UCLA; 13 females, 20-37 years of age). All the participants gave written informed consent, and the experiment was approved by the UCLA Human Subjects Protection Committee. Three participants were excluded from the analyses, due to either faulty data collection or technical problems with stimulus presentation materials, leaving 14 participants for the present analyses (10 females, 20-37 years of age).

\section{Procedure}

To assess individual differences in neural reactivities to discrepancy, the participants were scanned while performing an oddball task, a discrepancy detection task that has been shown to activate the dACC (Braver et al., 2001; Weissman et al., 2003). During this task, the participants were shown letters one at a time and were instructed to press a button whenever a letter other than X appeared; these non-X trials appeared infrequently (oddballs). Nonoddballs (X) were presented on $80 \%$ of the trials, and oddballs (non-X: C, L, $\mathrm{T}$, or $\mathrm{V}$ ) were presented on $20 \%$ of the trials. Oddball trials were never presented back to back. The participants viewed the stimuli through goggles connected to a Macintosh G3 desktop computer that presented the stimuli, using MacStim software (MacStim2, Version 2.6.1). In a rapid event-related design, the scan began and ended with a fixation crosshair presented for $12 \mathrm{sec}$. Following the initial crosshair, 3001 -sec trials were presented, each consisting of the presentation of a single letter. The participants responded using a button box that rested against their torso.

Five to 14 days after the participants had been scanned, in a separate experimental session, the participants completed self-report measures of neuroticism, extraversion (Eysenck Personality Questionnaire; S. B. G. Eysenck, \& H. J. Eysenck, 1975), and selfconsciousness (Self-Consciousness Scale: Fenigstein, Scheier, \& Buss, 1975) and then participated in an interoceptive accuracy task. For this task, the participants were told that they would complete a brief exercise task and would then be asked to report on how aroused they felt while actual measures of heart rate (HR) and blood pressure (BP) were taken. They were instructed to think of $0 \%$ as the amount of arousal that they felt immediately before exercising and $100 \%$ as the amount of arousal that they felt immediately after exercising. Before the exercise period, a baseline assessment of HR and BP was taken using an automatic BP monitor (Omron automatic blood pressure monitor, Model HEM-712C). Following the baseline reading, the participants stepped up and down on a 9-in. step for $1 \mathrm{~min}$. Immediately following the exercise period, measures of HR and BP and measures of perceived arousal were assessed simultaneously every $2 \mathrm{~min}$ for $8 \mathrm{~min}$ post-exercise.

An index of actual physiological arousal was computed by combining HR and systolic BP (SBP) measures at each of the five time points to create an index called the rate pressure product $[\mathrm{RPP}=$ $(\mathrm{HR} * \mathrm{SBP}) / 100 ;$ Pham, Taylor, \& Seeman, 2001]. This measure was used because it tends to be a gender-neutral measure of physiological arousal, due to the fact that women show increased HR whereas men show increased BP, in response to physiological challenge (Allen, Stoney, Owens, \& Matthews, 1993).

Interoceptive accuracy was assessed by computing the slopes of actual arousal and of perceived arousal scores over the five time points (immediately following exercise and then at 2, 4, 6, and 8 min postexercise) and then taking the absolute value of the difference in these slopes. Using slopes rather than arousal judgments of single time points circumvents the need to calibrate subjective and objective arousal measures to a common scale. For ease of interpretation, all the difference scores were multiplied by -1 , so that lower scores represent lower interoceptive accuracy and higher scores represent higher interoceptive accuracy.

\section{fMRI Data Acquisition and Data Analysis}

Data were acquired on a G.E. 3T full-body scanner with an upgrade for echo-planar imaging (EPI; Advanced NMR Systems). Head movements were restrained with foam padding and surgical tape placed across each participant's forehead. For each participant, a highresolution structural T2-weighted EPI volume (spin echo; TR = $4,000 \mathrm{msec}$; TE $=54 \mathrm{msec}$; matrix size, $128 \times 128 ; 26$ axial slices; $3.125-\mathrm{mm}$ in-plane resolution; $4 \mathrm{~mm}$ thick; skip, $1 \mathrm{~mm}$ ) was acquired coplanar with the functional scans. A functional scan was acquired (echo planar $\mathrm{T}_{2}{ }^{*}$-weighted gradient echo; $\mathrm{TR}=2,000 \mathrm{msec}, \mathrm{TE}=$ 
$25 \mathrm{msec}$, flip angle $=90^{\circ}$, matrix size $64 \times 64 ; 13$ axial slices; 3.125 $\mathrm{mm}$ in-plane resolution; $4 \mathrm{~mm}$ thick; skip, $1 \mathrm{~mm}$ ) spanning the entire ACC, lasting $5 \mathrm{~min} 24 \mathrm{sec}$.

The imaging data were analyzed using statistical parametric mapping (SPM'99; Wellcome Department of Cognitive Neurology, Institute of Neurology, London). Images for each participant were realigned to correct for head motion, slice-timed to account for time differences in slice acquisition, normalized into a standard stereotactic space, as defined by the Montreal Neurological Institute, and smoothed with an 8-mm Gaussian kernel, full width at half maximum. For each participant, individual trials were modeled as events, using a canonical hemodynamic response and its temporal derivative. Linear contrasts were employed to compare neural activity during oddball trials with neural activity during nonoddball trials.

To assess correlations between individual difference measures and neural activity, the participants' neuroticism, extraversion, selfconsciousness, and interoceptive accuracy scores were entered separately as regressors into a random effects group analysis comparing activations for oddball trials with activations for nonoddball trials. Peristimulus hemodynamic time courses were computed by identifying clusters of activations from the regression analyses and then applying a selective averaging procedure on a participant-byparticipant basis to these clusters (R. A. Poldrack, University of California, Los Angeles). Time courses were also adjusted for personality differences to create personality-weighted time courses, by multiplying each participant's time course by a normalized estimate of each of the participant's personality scores. Following this, personalityweighted time courses were averaged across participants. This yielded three group-averaged time courses for each cluster: one weighted by neuroticism scores, one weighted by extraversion scores, and one weighted by self-consciousness scores. The correction for multiple comparisons was carried out using an uncorrected $p$ value of .005 , combined with a cluster size threshold of 10 voxels.

\section{RESULTS}

\section{Behavioral Data}

Correlations between personality measures. Similar to previously reported correlations between these personality measures (Darvill, Johnson, \& Danko, 1992;
Trapnell \& Campbell, 1999), extraversion was significantly negatively correlated with self-consciousness $[r(14)=-.58, p<.05]$ and marginally negatively correlated with neuroticism $[r(14)=-.42, p=.13]$. In addition, neuroticism and self-consciousness were strongly positively correlated with each other $[r(14)=.77, p<$ $.01)$.

Neuroticism and interoceptive accuracy. Next, we examined interoceptive accuracy as a behavioral correlate of neuroticism. In order to assess interoceptive accuracy apart from arousal differences that might facilitate accurate detection of interoceptive changes, we first examined whether there was a relationship between actual physiological arousal levels following exercise and interoceptive accuracy scores. Individuals with a steeper decline in physiological arousal levels following exercise revealed a marginal tendency to be more accurate at reporting on arousal changes $[r(14)=-.46, p=.10]$. Consequently, we controlled for the slope in physiological arousal levels in the subsequent analyses involving interoceptive accuracy.

Replicating previous studies showing a relationship between neuroticism-related personality measures and interoceptive accuracy (Critchley et al., 2004; LudwickRosenthal \& Neufeld, 1985; Schandry, 1981; Stegen et al., 2001; Stewart et al., 2001; Sturges \& Goetsch, 1996), the behavioral results showed an association between selfreported neuroticism and interoceptive accuracy. The correlation between neuroticism and interoceptive accuracy, controlling for arousal levels, was marginally significant $[r(14)=.40, p=.08$; one-tailed for a priori prediction; see Figure 4A] and fell within the range of effect sizes observed in previous studies $(r \mathrm{~s}=.27-.66)$. Extraversion did not correlate with interoceptive accuracy $[r(14)=-.04, p>.30]$, nor did self-consciousness $[r(14)=.28, p>.30]$.

\section{Neuroticism}
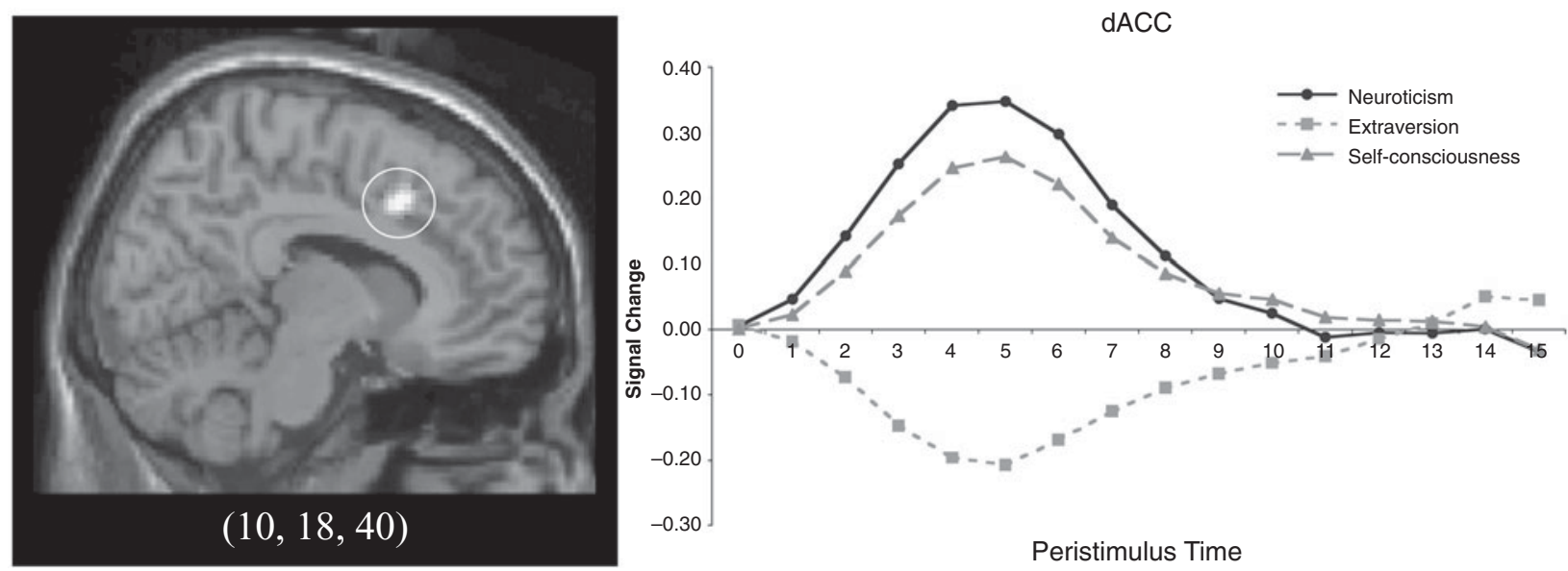

Figure 1. Dorsal anterior cingulate cortex (dACC) activation associated with neuroticism and time courses for the activity in this cluster weighted by neuroticism, extraversion, and self-consciousness scores. 


\section{Extraversion}
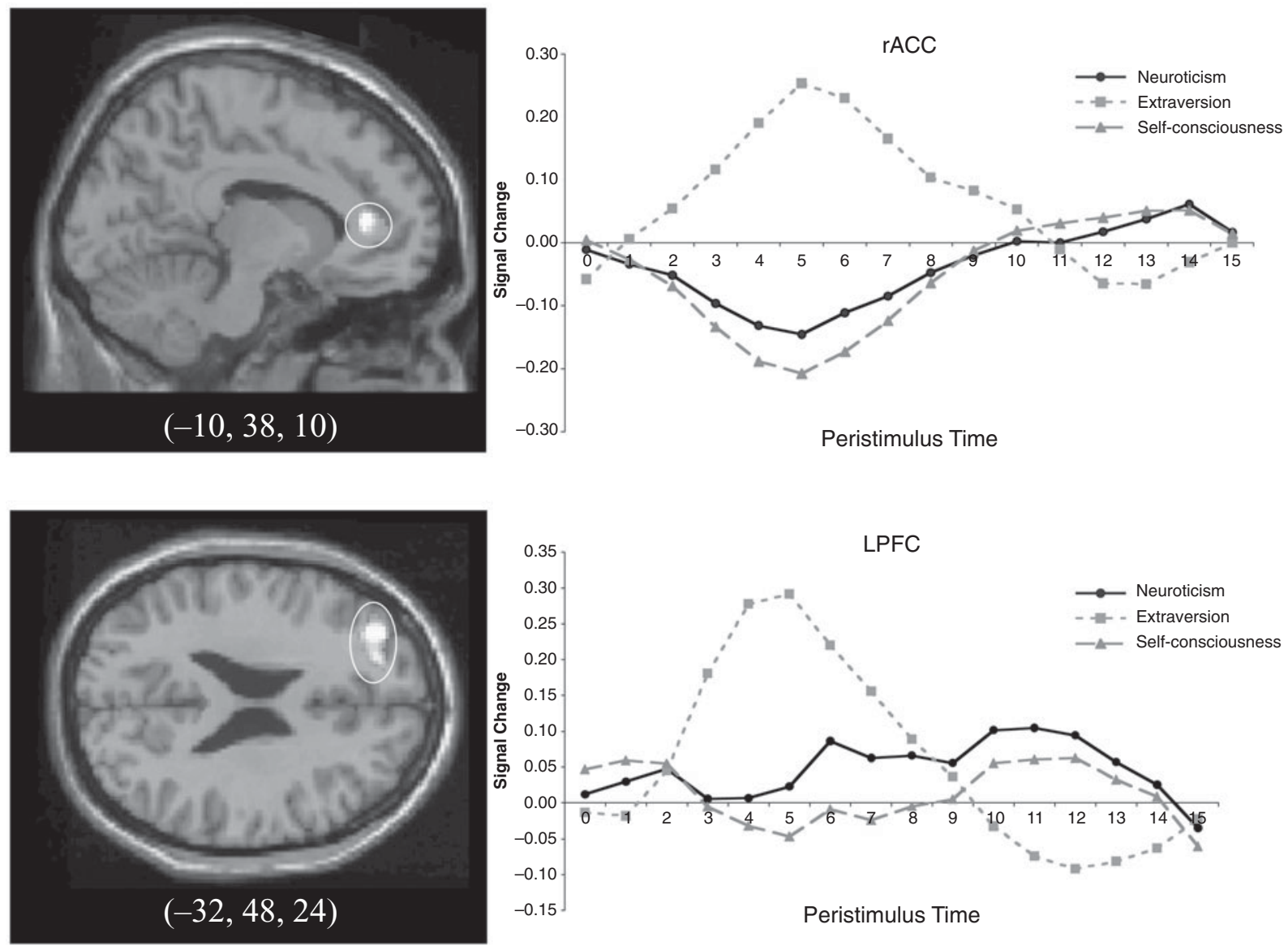

Figure 2. Rostral anterior cingulate cortex (rACC) and lateral prefrontal cortex (LPFC) activations associated with extraversion and time courses for the activity in each of these clusters weighted by neuroticism, extraversion, and self-consciousness scores.

\section{Neuroimaging Data}

Neuroticism and dACC reactivity. To examine whether self-reported neuroticism was associated with increased dACC reactivity to discrepancy detection, we examined correlations between self-reported neuroticism levels and $\mathrm{dACC}$ reactivity to oddball, relative to nonoddball, trials. As was predicted, self-reported neuroticism correlated positively with activity in the dACC $(r=.81, p<.001)$, the area typically associated with discrepancy detection in neuroimaging studies of cognitive control (Botvinick et al., 2001; see Figure 1). Neuroticism was also negatively correlated with activity in the $\operatorname{rACC}(r=-.74, p<.005$; see Tables 1 and 2$)$, replicating previous findings showing a negative correlation between neuroticism-related constructs and rACC activity (Bishop et al., 2004; J. R. Gray \& Braver, 2002). In addition, neuroticism correlated negatively with both LPFC and LPPC, areas that we hypothesized to be related to higher levels of extraversion (see Tables 1 and 2).
Due to the large correlation between neuroticism and self-consciousness $(r=.77)$, we conducted additional analyses to examine the unique relationship between self-reported neuroticism and neural activity by controlling for self-reported self-consciousness. To do this, selfconsciousness scores were first regressed out of neuroticism scores, using regression analyses in a standard statistical package. The unstandardized residuals (neuroticism controlling for self-consciousness) were then used as the regressor in a group analysis, which was carried out at a significance value of $p=.005$ and an extent threshold of 10 voxels. Neuroticism, controlling for selfconsciousness, remained significantly positively correlated with a region of the dACC more posterior to the original activation $(x=14, y=-6, z=50 ; t=4.47, r=.79$, $p<.005$ ) but was no longer significantly negatively correlated with rACC, LPFC, or LPPC activity. This suggests that neuroticism, as distinct from self-consciousness, may be primarily associated with dACC activity. However, due 
Table 1

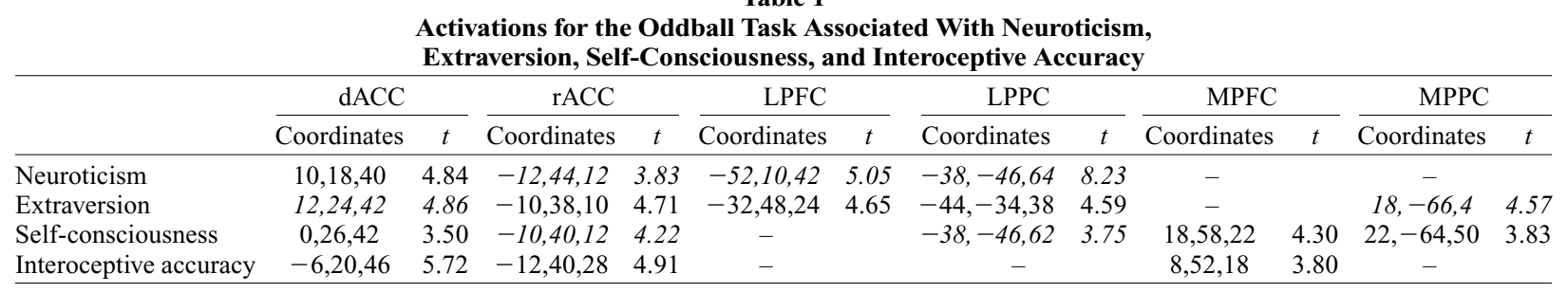

Note-Coordinates refer to the the Montreal Neurological Institute coordinate space. Roman numbers indicate positive correlations, and italicized numbers indicate negative correlations. dACC, dorsal anterior cingulate cortex; rACC, rostral anterior cingulate cortex; LPFC, lateral prefrontal cortex; LPPC, lateral posterior parietal cortex; MPFC, medial prefrontal cortex; MPPC, medial posterior parietal cortex.

to the high correlation between self-reported neuroticism and self-consciousness and the small sample size, it is difficult to draw strong conclusions from this analysis.

Extraversion and LPFC/LPPC activity. To investigate whether extraversion was related to LPFC and LPPC responses to discrepancy, we examined correlations between self-reported levels of extraversion and LPFC and LPPC activity during the oddball task. As was expected, extraversion correlated positively with left LPFC activity $(r=.80, p<.001)$ and with left LPPC activity $(r=.81$, $p<.001)$, when oddball were compared with nonoddball trials (see Figure 2). Extraversion was also positively correlated with $\mathrm{rACC}$ activity, replicating previous findings (Canli, Amin, Haas, Omura, \& Constable, 2004) and was negatively correlated with dACC and MPPC activity (see Tables 1 and 2).

Self-consciousness and MPFC/MPPC activity. To investigate whether self-consciousness was related to heightened MPFC and MPPC responses to discrepancy, we examined correlations between self-reported levels of self-consciousness and MPFC and MPPC activity during the oddball task. As was predicted, self-consciousness was associated with an increase in MPFC activity ( $r=$ $.78 ; p<.001)$ and with an increase in MPPC activity $(r=.74, p<.005)$ during oddball as compared with nonoddball, trials (see Figure 3 ). In addition, similar to the pattern found for neuroticism, self-consciousness was positively correlated with dACC activity and negatively correlated with rACC and LPPC activity (see Tables 1 and 2). Because responses to oddball trials were compared with those to nonoddball trials, the results should be interpreted as relative activations. This is especially important with respect to MPFC findings, since the MPFC is a region that is often more active at rest than during an active task (Gusnard \& Raichle, 2001; Kelley et al., 2002; cf. Iacoboni et al., 2004).

Again, due to the large correlation between selfconsciousness and neuroticism, we examined the correlation between self-consciousness and neural activity, controlling for neuroticism, using the same methodology as that used to exame the unique association between neuroticism and neural activity. Self-reported selfconsciousness, controlling for neuroticism, remained significantly positively correlated with MPFC activity $(x=$
$20, y=58, z=22 ; t=3.94, r=.75, p<.005)$ and significantly negatively correlated with rACC activity $(x=-8$, $y=38, z=14 ; t=4.27, r=-.77, p<.005)$. However, after controlling for neuroticism, self-consciousness was no longer significantly positively correlated with dACC or MPPC activity or negatively correlated with LPPC activity. These results suggest that self-consciousness may be associated primarily with increased MPFC activity and decreased rACC activity when discrepancy is detected. Again, these findings should be interpreted with caution, due to the small sample size.

dACC reactivity and interoceptive accuracy. The findings thus far have indicated that self-report measures are associated with neural reactivities that map onto different aspects of the TOTE unit model of control. However, an additional question in this study is whether neural reactivities are more powerful than self-report measures in predicting relevant behavioral outcome measures. In this study, we chose to compare self-reported neuroticism with dACC reactivity, a neural measure of neuroticism, with respect to their abilities to predict interoceptive accuracy. To do this, we first investigated the relationship between individual differences in AACC reactivity to the oddball task and interoceptive accuracy. We then compared the predictive ability of the neural and the self-report measures in explaining variance in interoceptive accuracy.

Because individuals with a steeper decline in physiological arousal levels after exercise were more accurate, we examined the correlation between neural activity and interoceptive accuracy, controlling for the slope in phys-

Table 2

Correlations Between Activations for the Oddball Task and Neuroticism, Extraversion, Self-Consciousness, and Interoceptive Accuracy

\begin{tabular}{lrrrrrc}
\hline & dACC & rACC & LPFC & LPPC & MPFC & MPPC \\
\hline Neuroticism & .81 & -.74 & -.82 & -.92 & - & - \\
Extraversion & -.81 & .81 & .80 & .81 & - & -.80 \\
Self-consciousness & .71 & -.77 & - & -.73 & .78 & .74 \\
Interoceptive accuracy & .86 & .82 & - & - & .74 & - \\
\hline
\end{tabular}

Note-dACC, dorsal anterior cingulate cortex; rACC, rostral anterior cingulate cortex; LPFC, lateral prefrontal cortex; LPPC, lateral posterior parietal cortex; MPFC, medial prefrontal cortex; MPPC, medial posterior parietal cortex. 


\section{Self-Consciousness}
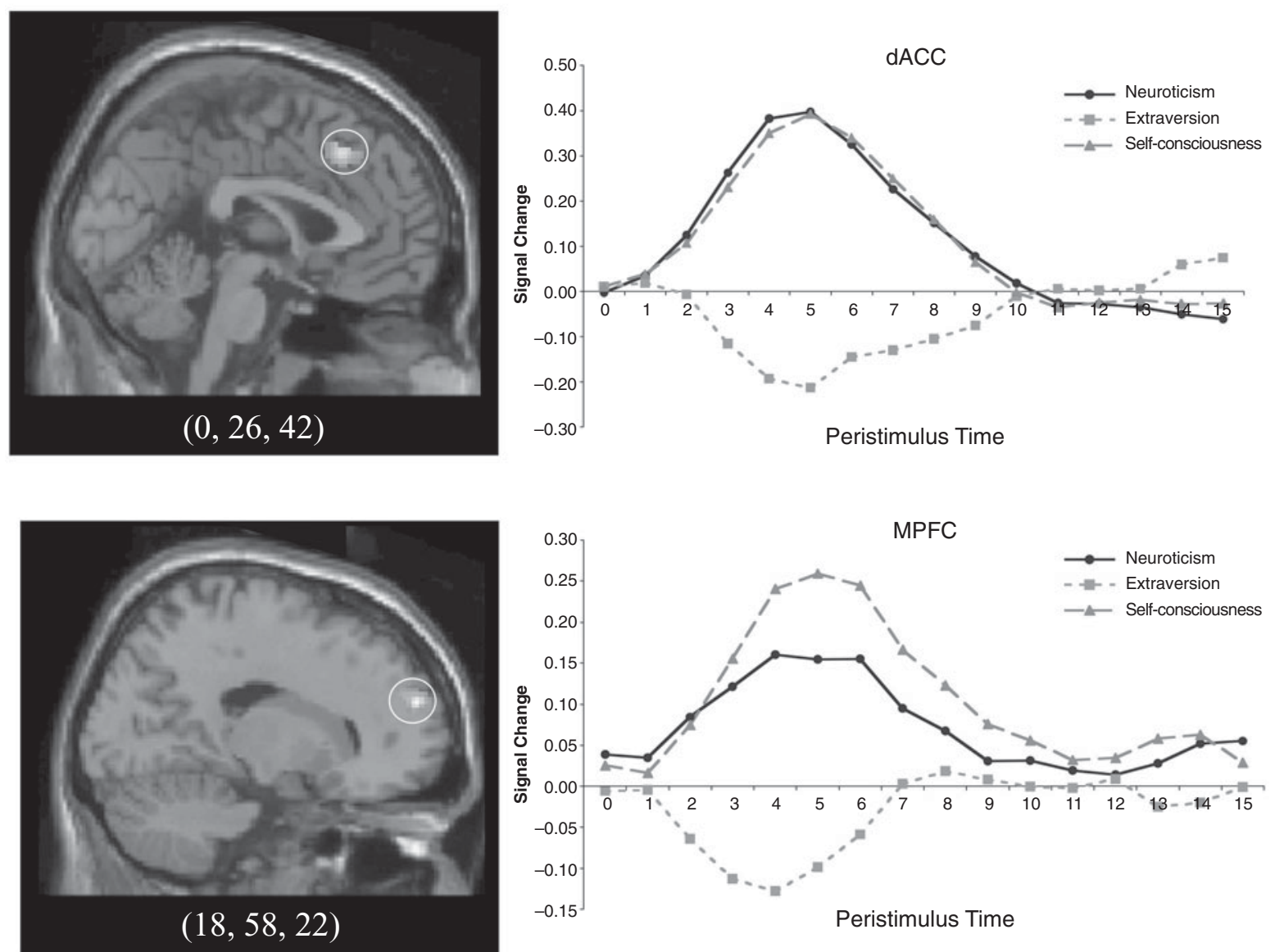

Figure 3. Dorsal anterior cingulate cortex (dACC) and medial prefrontal cortex (MPFC) activations associated with selfconsciousness and time courses for the activity in each of these clusters weighted by neuroticism, extraversion, and selfconsciousness scores.

iological arousal levels over time. As shown in Figure 4B, dACC activity $(x=-6, y=20, z=46)$ was strongly positively correlated with interoceptive accuracy $(r=$ $.86 ; p<.001)$. In addition, interoceptive accuracy was also positively correlated with rACC activity $(r=.82$, $p<.001)$ and with MPFC activity $(r=.74 ; p<.005$; see Tables 1 and 2).

To assess which neural region was most strongly associated with interoceptive accuracy, we computed partial correlations, examining the relationship between one neural region, controlling for another, and interoceptive accuracy. When the activity of the MPFC was controlled for, the region of dACC activity was still significantly associated with interoceptive accuracy $[r(14)=.67, p<.01]$. Likewise, when the activity of the rACC was controlled for, the region of dACC activity was still significantly associated with interoceptive accuracy $[r(14)=.56, p<$ $.05]$. However, after the activity of the dACC was controlled for, neither MPFC $[r(14)=.28, p=.36]$ nor rACC $[r(14)=.38, p=.20]$ activity was significantly associated with interoceptive accuracy. Thus, dACC activity seems to be most strongly related to interoceptive accuracy.

In terms of simple correlations, interoceptive accuracy was more strongly correlated with dACC reactivity $(r=.86)$ than with self-reported neuroticism $(r=.40)$. However, to examine whether AACC reactivity accounted for a significant amount of the variance in interoceptive accuracy over and above what was already accounted for by self-reported neuroticism and whether self-reported neuroticism accounted for a significant amount of the variance over and above what was accounted for by dACC reactivity, we conducted multiple regression analyses. Whereas dACC reactivity accounted for a significant amount of the variance over and above that which was accounted for by self-reported neuroticism $(F$ change $=$ 23.76, $p<.001$ ), self-reported neuroticism did not account for any additional variance over and above dACC reactivity $(F$ change $=0.1$, n.s. $)$. 

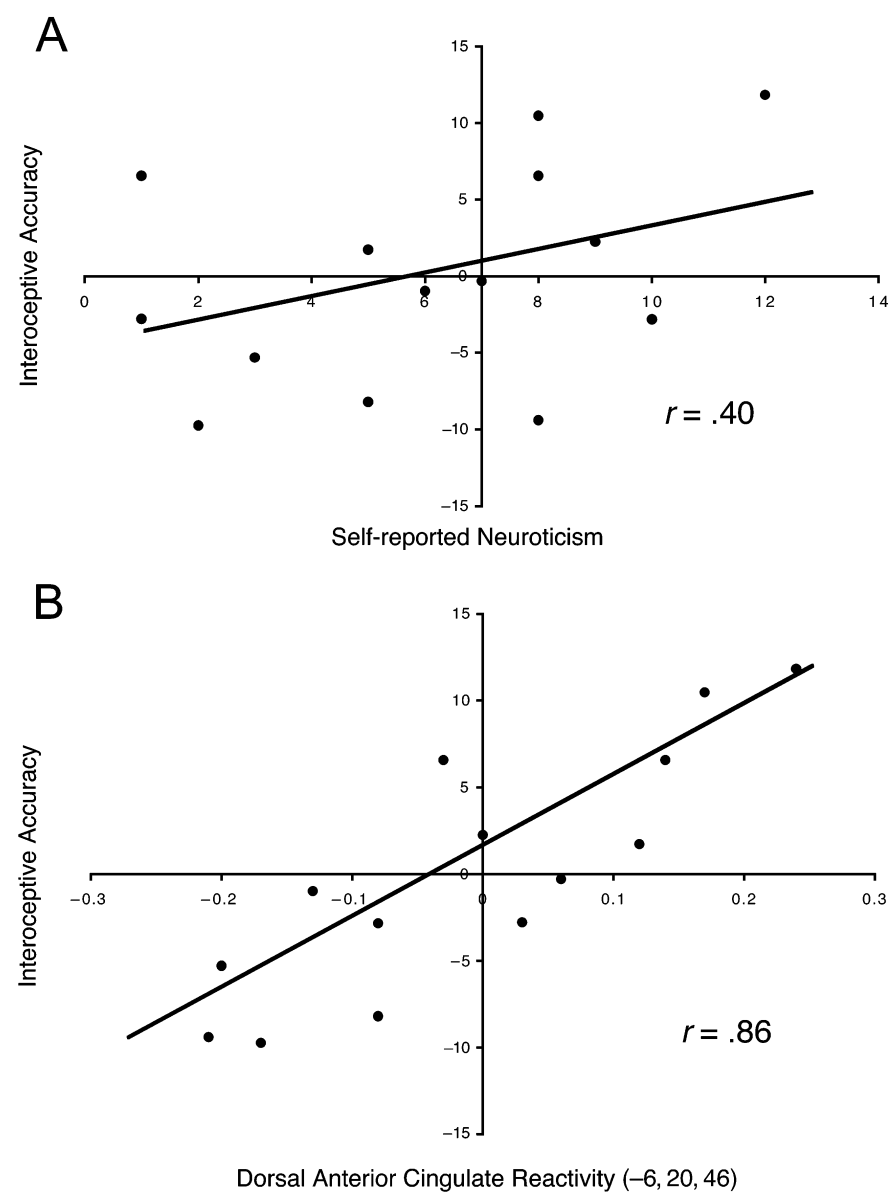

Figure 4. (A) Scatterplot showing self-reported neuroticism scores, plotted against interoceptive accuracy (controlling for arousal levels) across participants. (B) Scatterplot showing dorsal anterior cingulate cortex activations to oddball trials relative to nonoddball trials, plotted against interoceptive accuracy (controlling for arousal levels) across participants.

\section{DISCUSSION}

Personality is among the oldest areas of psychological theorizing, extending back at least to Galen's bodily humor taxonomy in ancient Greece (Kagan, 1994), and continues to be an area of great interest, appealing to anyone interested in understanding what makes each of us different from others, despite our common bond of humanity. However, even with this enduring and deep interest, personality research has been limited in several ways.

\section{Aristotelian Versus Galilean Approaches to Personality}

One limitation of personality research is that much of it has focused on categorizing personality traits, rather than on explaining them. Although public interest in personality is driven by identifying differences in experi- ence and behavior, a personality science must ultimately turn to the mechanisms responsible for these differences. Identifying neurotics as frequently experiencing negative affect and extraverts as happy and gregarious tells us what it means to be neurotic or extraverted and how to know one when we see one, but it does not identify the mechanisms contributing to these traits.

Lewin (1935) referred to this kind of taxonomic approach as Aristotelian science and suggested that powerful insights almost always come instead from Galilean science. Aristotelian science refers to the careful observation and categorization of similar phenotypes. Galilean science, on the other hand, is the empirical study of the unobserved causes of that which is observed. We have suggested that individual differences in the components of the neural structures supporting controlled processing may be some of the unobserved causes of the observable and experiential aspects of personality. On the basis of previous 
cognitive neuroscience investigations, we posited that the dACC constitutes the test component of a TOTE mechanism that triggers top-down control. In addition, we posited two operation components of a TOTE mechanism, triggered by the test component, that would actually instantiate the controlled processes: a lateral frontoparietal network (LPFC/LPPC) identified in numerous studies of cognitive control and a medial frontoparietal network (MPFC/MPPC) identified in studies of selffocused or self-reflective states.

We found that each of the three personality dimensions correlated strongly with a subset of these control mechanisms. Neuroticism was positively correlated with the test component in the dACC, whereas extraversion was positively correlated with the lateral operation network (LPFC/LPPC) and self-consciousness was positively correlated with the medial operation network (MPFC/MPPC). In addition, extraversion and self-consciousness were each associated with a test mechanism as well. Selfconsciousness, not surprisingly given its high correlation with neuroticism, was positively associated with dACC activity, and extraversion was positively associated with rACC activity.

The finding that neuroticism was positively correlated with dACC activity to discrepancy is in line with J. A. Gray's (1982) original hypothesis that anxiety is the result of a more sensitive neural comparator, which detects discrepancies between the actual and the expected states of the world. Although Gray proposed that this neural comparator was of a "septo-hippocampal" origin, the similarity between the function of J. A. Gray's comparator and the function of the AACC is unmistakable and lends support to J. A. Gray's original conceptualization of anxiety. In addition, the finding relating neuroticism to dACC reactivity builds on previous neuroimaging studies of neuroticism in which the relationship between neuroticism and amygdala reactivity has primarily been investigated (Canli et al., 2001).

That extraversion was positively correlated with the rACC, rather than with the dACC, is consistent with previous work by J. R. Gray and Braver (2002) and by Canli et al. (2004); however, there is little previous work suggesting that the $\mathrm{rACC}$ can serve as a trigger indicating the need for controlled processing. That both ACC regions are involved in responses to discrepancy is an important finding, although an understanding of the conditions under which the AACC and the rACC would respond to discrepancies requires further research (Eisenberger \& Lieberman, 2004).

A major limitation of the present study is the small sample size. Given the high correlation between selfreport measures of neuroticism and self-consciousness, it is difficult to clearly disentangle the specific neural activity relevant to each. Future studies should include a greater number of participants, in order to more clearly dissociate the relationship between each of these personality measures with respect to neural reactivities to discrepancy detection.

\section{Lateral and Medial Control Networks}

Before continuing with the implications of the present findings for personality research, it is worth taking a moment to consider the implications of this study for the cognitive neuroscience of control. We started from a welldefined control network involving the AACC, LPFC, and LPPC. We also examined the MPFC and MPPC, because of their prior relationships with self-focused states of attention. This medial frontoparietal network is not typically observed in cognitive neuroscience studies of controlled processing. Nevertheless, within the social psychological literature, there is a history of relating self-focused attention to controlled processing (Carver \& Scheier, 1981; Duval \& Wicklund, 1972; Mead, 1934). However, unlike other studies in which the medial frontoparietal network has been activated by inducing self-focused states, in the present study, use of the oddball paradigm did not require or obviously promote self-focused attention. It appears that for individuals high in self-consciousness, this network may be activated by dACC activity, regardless of whether self-focused attention is needed. In other words, having a strong connection between the $\mathrm{AACC}$ and the MPFC may be part of the basis for being self-conscious, such that triggers for cognitive control lead to selffocused attention.

These results suggest that there are at least two kinds of control that can be set in motion by discrepancy detection and that people with different personalities may rely on these two types of control to varying degrees. On the basis of our findings and in accord with previous work contrasting MPFC and LPFC functions (Crosson et al., 2001; Ochsner et al., 2004), it would seem that the lateral network is activated when one exerts task-focused controlled processes that are directed at regulating one's behavior in the context of the immediate environment. The medial network, on the other hand, may be activated when the detection of discrepancy leads to introspection about whether one is capable of responding effectively to current task demands or to consider the implications of one's current task performance for a more global selfevaluation. Such self-evaluative processes may allow an individual to decide whether persistence is likely to be rewarded or not, without immediately leading to any behavioral changes. Unfortunately, a dispositional tendency to question one's own abilities may lead to anxiety, rumination, and self-doubt-all documented downsides to self-focused attention (Mor \& Winquist, 2002).

The present study also raises important issues about how social psychologists conceptualize the relationship between cognitive load and self-control. Numerous behavioral studies have demonstrated that performing a cognitive task (i.e., a working memory task) concurrently with or prior to a task that requires self-reflection or selfcontrol impairs performance on the self-relevant task (Baumeister, Bratslavsky, Muraven, \& Tice, 1998; Heatherton \& Baumeister, 1991). It is a cornerstone of social cognition and dual-process models that there is a common pool of controlled processing resources for which 
self, social, and cognitive processes compete. The present results suggest that this account might not be accurate, since internally focused and externally focused controlled processes appear to be distinct. However, the finding that cognitive control interferes with self-focused control can be understood, not as a direct competition for the same pool of controlled processing resources, but as a result of inhibition between the medial and the lateral networks. McKiernan and colleagues (McKiernan, Kaufman, Kucera-Thompson, \& Binder, 2003) have shown, in an elegant parametric fMRI study, that increasing LPFC activity leads to systematic deactivations of the MPFC. Thus, one's ability to simultaneously engage in externally and internally focused controlled processing may depend on individual differences in the inhibitory connections between the medial and the lateral networks, rather than on the size of a common resource pool.

\section{Improving on Self-Report}

Returning to personality research, we believe that the present research identifies an additional factor that has contributed to limitations of personality research. Although many of the most prominent theories of personality have posited an underlying neural signature that gives rise to the personality construct of interest (H. J. Eysenck, 1967; J. A. Gray, 1982), personality researchers have been restricted to assessing the secondary outcomes of these neural reactivities through self-reports. This leaves personality researchers playing a conceptual game of "telephone," the children's game in which a word or phrase is slowly degraded as it is whispered from one child to the next. Self-reports can be similarly described as the partially degraded assessment of the cognitive, behavioral, and affective consequences of the primary neural difference of interest. Indeed, this reliance on self-report measures may partially explain the rather modest correlation between self-reported personality measures and the behavioral measures that these constructs are intended to predict (Matthews \& Gilliland, 1999).

The present study indicates that it is possible to assess the neural activity associated with specific personality dimensions reasonably well and, perhaps, considerably better than self-report assessments of the same dimensions (see also J. R. Gray \& Braver, 2002). In the present study, the first neuroimaging study to include an outcome measure against which both self-reported and neurally assessed neuroticism could be compared, it was found that dACC reactivity was a substantially better predictor of interoceptive accuracy than self-reported neuroticism was, accounting for nearly five times the variance in interoceptive accuracy (74\% vs. 16\%). With the utilization of these types of methods, future personality research may have the potential to account for a substantially larger portion of the variation in human experience and behavior than has been possible with selfreport measures alone.

\section{REFERENCES}

Allen, M. T., Stoney, C. M., Owens, J. F., \& Matthews, K. A. (1993). Hemodynamic adjustments to laboratory stress: The influence of gender and personality. Psychosomatic Medicine, 55, 505-517.

Ardekani, B. A., Choi, S. J., Hossen-Zadeh, G., Porjesz, B., Tanabe, J. L., Lim, K. O., Bilder, R., Helpern, J. A., \& Begleiter, H. (2002). Functional magnetic resonance imaging of brain activity in the visual oddball task. Cognitive Brain Research, 14, 347-356.

Asmundson, G. J. G., Norton, G. R., Wilson, K. G., \& SANDler, L. S. (1994). Subjective symptoms and cardiac reactivity to brief hyperventilation in individuals with high anxiety sensitivity. Behaviour Research \& Therapy, 32, 237-241.

Baumeister, R. F., Bratslavsky, E., Muraven, M., \& Tice, D. M. (1998). Ego depletion: Is the active self a limited resource? Journal of Personality \& Social Psychology, 74, 1252-1265.

Bishop, S., Duncan, J., Brett, M., \& Lawrence, A. (2004). Prefrontal cortical function and anxiety: Controlling attention to threat-related stimuli. Nature Neuroscience, 7, 184-188.

Botvinick, M. M., Braver, T. S., Barch, D. M., Carter, C. S., \& Cohen, J. D. (2001). Conflict monitoring and cognitive control. Psychological Review, 108, 624-652.

Botvinick, M. M., Cohen, J. D., \& Carter, C. S. (2004). Conflict monitoring and anterior cingulate cortex: An update. Trends in $\mathrm{Cog}$ nitive Sciences, 8, 539-546.

Braver, T. S., Barch, D. M., Gray, J. R., Molfese, D. L., \& SnyDER, A. (2001). Anterior cingulate cortex and response conflict: Effects of frequency, inhibition and errors. Cerebral Cortex, 11, 825-836.

Breiter, H. C., Rauch, S. L., Kwong, K. K., Baker, J. R., WeissKoff, R. M., Kennedy, D. N., Kendrick, A. D., Davis, T. L., Jiang, A., Cohen, M. S., Stern, C. E., Belliveau, J. W., Baer, L., O'Sullivan, R. L., Savage, C. R., Jenike, M. A., \& Rosen, B. R. (1996). Functional magnetic resonance imaging of symptom provocation in obsessive-compulsive disorder. Archives of General Psychiatry, 53, 595-606.

Bunge, S. A., Hazeltine, E., Scanlon, M. D., Rosen, A. C., \& Gabrieli, J. D. (2002). Dissociable contributions of prefrontal and parietal cortices to response selection. NeuroImage, 17, 1562-1571.

Bystritsky, A., Pontillo, D., Powers, M., SabB, F. W., Craske, M. G., $\&$ BookHEIMER, S. Y. (2001). Functional MRI changes during panic anticipation and imagery exposure. NeuroReport, 12, 3953-3957.

Canli, T., Amin, Z., HaAs, B., Omura, K., \& Constable, R. T. (2004). A double dissociation between mood states and personality traits in the anterior cingulate. Behavioral Neuroscience, 118, 897-904.

Canli, T., Zhao, Z., Desmond, J. E., Kang, E., Gross, J., \& GabRIELI, J. D. (2001). An fMRI study of personality influences on brain reactivity to emotional stimuli. Behavioral Neuroscience, 115, 33-42.

Carter, C. S., Braver, T. S., Barch, D. M., Botvinick, M. M., Noll, D., \& Cohen J. D. (1998). Anterior cingulate cortex, error detection and the online monitoring of performance. Science, 288, 1835-1838.

Carter, C. S., Macdonald, A. M., Botvinick, M., Ross, L. L., Stenger, V. A., Noll, D., \& Cohen, J. D. (2000). Parsing executive processes: Strategic versus evaluative functions of the anterior cingulate cortex. Proceedings of the National Academy of Sciences, 97, 1944-1948.

CARVER, C. S., \& SCHEIER, M. F. (1981). Attention and self-regulation: A control theory approach to human behavior. New York: SpringerVerlag.

CARver, C. S., \& Scheier, M. F. (1990). Origins and functions of positive and negative affect: A control-process view. Psychological Review, 97, 19-35.

Costa, P. T., \& McCrae, R. R. (1980). Influence of extraversion and neuroticism on subjective well-being: Happy and unhappy people. Journal of Personality \& Social Psychology, 38, 668-678.

Critchley H. D., Wiens, S., Rothstein, P., Ohman, A., \& Dolan, R. J. (2004). Neural systems supporting interoceptive awareness. Nature Neuroscience, 7, 189-195.

Crosson, B., Sadek, J. R., Maron, L., Gokcay, D., Mor, C. M., Auer- 
back, E. J., Freeman, A. J., Leonard, C. M., \& Briggs, R. W. (2001). Relative shift in activity from medial to lateral frontal cortex during internally versus externally guided word generation. Journal of Cognitive Neuroscience, 13, 272-283.

Darvill, T. J., Johnson, R. C., \& Danko, G. P. (1992). Personality correlates of public and private self consciousness. Personality \& Individual Differences, 13, 383-384.

Duval, S., \& WickLund, R. A. (1972). A theory of objective selfawareness. New York: Academic Press.

Ehlers, A., \& Breuer, P. (1992). Increased cardiac awareness in panic disorder. Journal of Abnormal Psychology, 101, 371-382.

EisenberGer, N. I., \& Lieberman, M. D. (2004). Why rejection hurts: A common neural alarm system for physical and social pain. Trends in Cognitive Sciences, 8, 294-300.

EYSENCK, H. J. (1967). The biological basis of personality. Springfield, IL: Thomas.

Eysenck, S. B. G., \& Eysenck, H. J. (1975). Manual of the Eysenck Personality Questionnaire. London: Hodder \& Stoughton.

Fenigstein, A., Scheier, M. F., \& Buss, A. H. (1975). Public and private self-consciousness: Assessment and theory. Journal of Consulting \& Clinical Psychology, 43, 522-527.

GraY, J. A. (1981). A critique of Eysenck's theory of personality. In H. J. Eysenck (Ed.), A model for personality (pp. 272-273). New York: Springer-Verlag.

Gray, J. A. (1982). The neuropsychology of anxiety: An enquiry into the functions of the septo-hippocampal system. Oxford: Oxford University Press.

Gray, J. R., \& Braver, T. S. (2002). Personality predicts workingmemory-related activation in the caudal anterior cingulate cortex. Cognitive, Affective, \& Behavioral Neuroscience, 2, 64-75.

Gusnard, D. A., Akbudak, E., Shulman, G. L., \& Raichle, M. E. (2001). Medial prefrontal cortex and self-referential mental activity: Relation to a default mode of brain function. Proceedings of the $\mathrm{Na}$ tional Academy of Sciences, 98, 4259-4264.

Gusnard, D. A., \& RaIChle, M. E. (2001). Searching for a baseline: Functional imaging and the resting human brain. Nature Reviews Neuroscience, 2, 685-694.

Heatherton, T. F., \& Baumeister, R. F. (1991). Binge eating as escape from self-awareness. Psychological Bulletin, 110, 86-108.

HigGins, E. T. (1987). Self-discrepancy: A theory relating self and affect. Psychological Review, 94, 319-340.

Iacoboni, M., Lieberman, M. D., Knowlton, B. J., MolnarSzakacs, I., Moritz, M., Throop, C. J., \& Fiske, A. P. (2004). Watching social interactions produces dorsomedial prefrontal and medial parietal BOLD fMRI signal increases compared to a resting baseline. NeuroImage, 21, 1167-1173.

Johnson, S. C., Baxter, L. C., Wilder, L. S., Pipe, J. G., Heiserman, J. E., \& PRigatano, G. P. (2002). Neural correlates of self-reflection. Brain, 125, 1808-1814.

KaGan J. (1994). Galen's prophecy: Temperament in human nature. New York: Basic Books.

Kampe, K. K. W., Frith, C. D., \& Frith, U. (2003). "Hey John": Signals conveying communicative intention toward the self activate brain regions associated with "mentalizing," regardless of modality. Journal of Neuroscience, 23, 5258-5263.

Kelley, W. M., Macrae, C. N., Wyland, C. L., Caglar, S., Inati, S., \& Heatherton, T. F. (2002). Finding the self? An event-related fMRI study. Journal of Cognitive Neuroscience, 14, 785-794.

Kimbrell, T. A., George, M. S., Parekh, P. I., Ketter, T. A., PoDell, D. M., Danielson, A. L., Repella, J. D., Benson, B. E., Willis, M. W., Herscovitch, P., \& Post, R. M. (1999). Regional brain activity during transient self-induced anxiety and anger in healthy adults. Biological Psychiatry, 46, 454-465.

Kircher, T. T. J., Senior, C., Phillips, M. L., Rabe-Hesketh, S., Benson, P. J, Bullmore, E. T., Brammer, M., Simmons, A., BarTELS, M., \& DaVID, A. S. (2001). Recognizing one's own face. Cognition, 78, 1-15.

KJAER, K. W., NowAK, M., \& Lou, H. C. (2002). Reflective self-awareness and conscious states: PET evidence for a common midline parietofrontal core. NeuroImage, 17, 1080-1086.

Kumari, V., fFytche, D. H., Williams, S. C., \& Gray, J. A. (2004).
Personality predicts brain responses to cognitive demands. Journal of Neuroscience, 24, 10636-10641.

LaZARUS, R. S. (1991). Progress on a cognitive-motivational-relational theory of emotion. American Psychologist, 46, 819-834.

Lewin, K. (1935). The conflict between Aristotelian and Galileian modes of thought in contemporary psychology. In W. Knows (Ed.). A dynamic theory of personality: Selected papers (pp. 1-42). New York: McGraw-Hill.

LieberMan, M. D. (2000). Introversion and working memory: Central executive differences. Personality \& Individual Differences, 28, 479-486.

Lieberman, M. D., \& Rosenthal, R. (2001). Why introverts can't always tell who likes them: Multi-tasking and nonverbal decoding. Journal of Personality \& Social Psychology, 80, 294-310.

Ludwick-Rosenthal, R., \& Neufeld, R. W. J. (1985). Heart beat interoception: A study of individual differences. International Journal of Psychophysiology, 3, 57-65.

MandLeR, G. (1975). Mind and emotion. New York: Wiley.

Matthews, G., \& Gilliland, K. (1999). The personality theories of H. J. Eysenck and J. A. Gray: A comparative review. Personality \& Individual Differences, 26, 583-626.

McKiernan, K. A., Kaufman, J. N., Kucera-Thompson, J., \& BINDER, J. R. (2003). A parametric manipulation of factors affecting task-induced deactivation in functional neuroimaging. Journal of Cognitive Neuroscience, 15, 394-408.

Mead, G. H. (1934). Mind, self, and society. Chicago: University of Chicago Press.

Miller, G. A., Galanter, E., \& Pribram, K. H. (1960). Plans and the structure of behavior. New York: Holt.

Mor, N., \& WINQUIST, J. (2002). Self-focused attention and negative affect: A meta-analysis. Psychological Bulletin, 128, 638-662.

Nisbett, R. E., \& WiLson, T. D. (1977). Telling more than we can know: Verbal reports on mental processes. Psychological Review, 84, 231-259.

Ochsner, K. N., Ray, R. D., Cooper, J. C., Roberston, E. R., ChoPRA, S., Gabrieli, J. D. E., \& Gross, J. J. (2004). For better or for worse: Neural systems supporting the cognitive down- and up-regulation of negative emotion. NeuroImage, 23, 483-499.

Osuch, E. A., Ketter, T. A., Kimbrell, T. A., George, M. S., Benson, B. E., Willis, M. W., Herscovitch, P., \& Post, R. M. (2000). Regional cerebral metabolism associated with anxiety symptoms in affective disorder patients. Biological Psychiatry, 48, 1020-1023.

Pham, L. B., Taylor, S. E., \& Seeman, T. E. (2001). Effects of environmental predictability and personal mastery on self-regulatory and physiological processes. Personality \& Social Psychology Bulletin, 27, 611-620.

SCHANDRY, R. (1981). Heart beat perception and emotional experience. Psychophysiology, 18, 483-488.

Shallice, T. (1988). From neuropsychology to mental structure. New York: Cambridge University Press.

Shostak, B. B., \& Peterson, R. A. (1990). Effects of anxiety sensitivity on emotional response to a stress task. Behaviour Research \& Therapy, 28, 513-521.

SMith, E. E., \& Jonides, J. (1999). Storage and executive processes in the frontal lobes. Science, 283, 1657-1661.

Smith, E. R., \& Miller, F. D. (1978). Limits on perception of cognitive processes: A reply to Nisbett and Wilson. Psychological Review, $\mathbf{8 5}, 355-362$.

Stegen, K., Van Diest, I., Van de Woestijne, K. P., \& Van den BERGH, O. (2001). Do persons with negative affect have an attentional bias to bodily sensations? Cognition and Emotion, 15, 813-829.

Stewart, S. H., Buffett-Jerrott, S. E., \& Kokaram, R. (2001). Heartbeat awareness and heart rate reactivity in anxiety sensitivity: A further investigation. Anxiety Disorders, 15, 535-553.

Sturges, L. V., \& Goetsch, V. L. (1996). Psychophysiological reactivity and heartbeat awareness in anxiety sensitivity. Journal of Anxiety Disorders, 10, 283-294.

Trapnell, P. D., \& Campbell, J. D. (1999). Private self-consciousness and the five-factor model of personality: Distinguishing rumination from reflection. Journal of Personality \& Social Psychology, 76, 284304.

Ursu, S., Stenger, V. A., Shear, M. K., Jones, M. R., \& Carter, C. S. 
(2003). Overactive action monitoring in obsessive-compulsive disorder: Evidence from functional magnetic resonance imaging. Psychological Science, 14, 347-353.

VALlacher, R. R., \& Wegner, D. M. (1987). What do people think they're doing? Action identification and human behavior. Psychological Review, 94, 3-15.

Watson, D., \& Pennebaker, J. W. (1989). Health complaints, stress, and distress: Exploring the central role of negative affectivity. Psychological Review, 96, 234-254.

WEINER, N. (1948). Cybernetics: Control and communication in the animal and the machine. Cambridge, MA: MIT Press.

Weissman, D. H., Giesbrecht, B., Song, A. W., Mangun, G. R., \& WoLDORFF, M. G. (2003). Conflict monitoring in the human anterior cingulate cortex during selective attention to global and local object features. NeuroImage, 19, 1361-1368.

\section{NOTE}

1. J. A. Gray $(1981,1982)$ used the term anxiety to refer to a personality construct that is similar to and often used interchangeably with neuroticism. Thus, when we use the term anxiety here, we are not referring to clinical forms of anxiety. However, to avoid any possible confusion, we have chosen to use the term neuroticism rather than anxiety to refer to this personality construct since the term neuroticism is less easily confused with clinical forms of anxiety.

(Manuscript received October 1, 2004; revision accepted for publication March 12, 2005.) 